\title{
Design and Performance Evaluation of Cooperative Retransmission Scheme for Reliable Multicast Services in Cellular Controlled P2P Networks
}

\author{
Zhang, Qi; Fitzek, F.H.P.; Iversen, Villy Bæk
}

Published in:

18th Annual IEEE International Sympositum on Personal, Indoor and Mobile Radio Communications

Link to article, DOI:

10.1109/PIMRC.2007.4394773

Publication date:

2007

Document Version

Publisher's PDF, also known as Version of record

Link back to DTU Orbit

Citation (APA):

Zhang, Q., Fitzek, F. H. P., \& Iversen, V. B. (2007). Design and Performance Evaluation of Cooperative Retransmission Scheme for Reliable Multicast Services in Cellular Controlled P2P Networks. In 18th Annual IEEE International Sympositum on Personal, Indoor and Mobile Radio Communications IEEE. https://doi.org/10.1109/PIMRC.2007.4394773

\section{General rights}

Copyright and moral rights for the publications made accessible in the public portal are retained by the authors and/or other copyright owners and it is a condition of accessing publications that users recognise and abide by the legal requirements associated with these rights.

- Users may download and print one copy of any publication from the public portal for the purpose of private study or research.

- You may not further distribute the material or use it for any profit-making activity or commercial gain

- You may freely distribute the URL identifying the publication in the public portal 


\section{DESIGN AND PERFORMANCE EVALUATION OF COOPERATIVE RETRANSMISSION SCHEME FOR RELIABLE MULTICAST SERVICES IN CELLULAR CONTROLLED P2P NETWORKS}

\author{
Qi Zhang \\ Department of Communication, \\ Optics \& Materials \\ Technical University of Denmark \\ Lyngby, Denmark
}

\author{
Frank H.P. Fitzek \\ Department of Communications \\ Technology \\ Aalborg University \\ Aalborg, Denmark
}

\author{
Villy B. Iversen \\ Department of Communication, \\ Optics \& Materials \\ Technical University of Denmark \\ Lyngby, Denmark
}

\begin{abstract}
Reliable multicast applications such as software distribution, data distribution and replication and mailing list delivery, etc. [1] are getting more and more interests from network and service providers. The conventional error/loss recovery schemes are not efficient when they are applied to multicast scenarios in wireless networks. The reason lies in the unreliable wireless channel, the limited wireless bandwidth and resource, the battery powered wireless devices, and others. To have an effective error/loss recovery scheme for reliable multicast in wireless networks, we advocate a new communication architecture. It is referred to as cellular controlled peer-topeer network [2], where the mobile devices communicate directly with each other to perform cooperative retransmissions using their short-range communication capabilities in addition to their cellular links. Based on the cooperative architecture a novel retransmission scheme is proposed exploiting the shortrange retransmission in this paper. The state of the art, the non-cooperative error recovery schemes (e.g., ARQ, Layered FEC and Integrated FEC II) and the proposed scheme are compared with each other in terms of energy consumption to show the benefit of the newly introduced scheme.
\end{abstract}

\section{INTRODUCTION}

Multicast communication has been identified as an effective way to disseminate information to a potential group of receivers [3] sharing the same service interest. Many data dissemination applications such as software distribution, data distribution and replication and mailing list delivery, etc. [1] require reliable multicast. It has received many researchers' interests and attentions on reliable multicast in the previous years $[1,3,4,5]$.

So far automatic repeat request (ARQ) and forward error correction (FEC) schemes are widely used for error/loss recovery. Pure ARQ has scalability issues such as implosion and exposure [6]. And pure FEC can not provide full reliability [4]. Combining ARQ with FEC (i.e., Hybrid ARQ I \& II) [7, 8, 9] can achieve better performance. But [4] proofed that HARQ II i.e., integrated FEC II can not always outperform pure ARQ and pure FEC schemes, specially if the receivers have heterogeneous packet loss probabilities, the performance is almost solely determined by the receivers with high loss rate, even though the fraction of high-loss receivers is very small.

The essential reason of integrated FEC performance degra- dation in wireless networks is that the sender always transmits the maximum number of parity packets needed by any receiver. So the channel heterogeneity in wireless networks limits the achievable performance. Moreover, if a radio path between an access point (AP) and a mobile device is greatly deteriorated by the instantaneous channel conditions, the mobile device can not effectively help itself out by requesting retransmissions. The other mobile devices suffer extra energy consumption because of receiving useless parity packets. Additionally, the packet latency is increased consequently. Although some modified strategies [4] proposed by stopping parities reception once one mobile device has enough parities, network bandwidth is wasted anyway. Furthermore, integrated FEC also has high processing load for coding and decoding.

Therefore, more efficient error/loss recovery solution for reliable multicast in wireless networks is worth investigating. In this paper, we propose one feasible solution called cooperative retransmission strategy. In this strategy, the mobile devices can recover error by cooperative retransmission with each others' proximity over the short-range link based on cellular controlled peer-to-peer (CCP2P) network architecture [2]. There are so-called "cooperative" multicast proposed in [10], which is used for ad-hoc sensor network. Hence, these two strategies are totally different.

The proposed solution can effectively save energy consumption because it reduces the average number of transmissions to receive a packet reliably at all the receivers. Consequently it can reduce retransmission delay and improve bandwidth utilization. In this paper, we compare the energy consumption of the cooperative retransmission scheme with the noncooperative schemes. The corresponding delay reduction and bandwidth utilization improvements are very obvious due to the reduction of the average retransmission times.

\section{NON-COOPERATIVE ERROR RECOVERY Strategies}

In the non-cooperative error recovery schemes the mobile device (MD) can only communicate with the access point (AP). This section presents the ARQ scheme and two FEC schemes of which energy consumption is derived.

\section{A. ARQ Scheme}

The ARQ scheme is widely used in many multicast protocols such as MTP (Multicast Transport Protocols), AFDP (Adaptive File Distribution Protocol), SRM (Scalable Reliable Multicast) and TMTP (Tree-based Multicast Transport Protocol) [11]. All 
these protocols have implosion and exposure issues. Implosion is resulted from the duplicate NACKs from many receivers. It might swamp the sender and the network, even the other receivers. Exposure occurs when the retransmitted packets are delivered to those receivers who did not lose the packets. Both implosion and exposure are fatal impediments for multicasting in wireless networks.

In this subsection, we use SRM (Scalable Reliable Multicast) protocol as one optimal example of ARQ scheme. In the SRM protocol the sender can suppress the NACKs from the different receivers that lost the same packet [11]. The energy consumption for a reliable packet reception is used as scheme evaluation criteria. The idea of energy calculation is: in order to have a packet reliably received, the mobile device can stay in three possible states (i.e., reception, transmission and idle states with different power level.) The mobile device is in reception state when it receives original or the retransmitted packets from AP. It switches to transmission state when it requests retransmission. It stays in idle state to save energy when other mobile devices are sending retransmission requests. So the energy consumption is the power level multiplying the duration that the mobile device staying at the corresponding power level.

We define:

- $P_{c, r x}, P_{c, t x}, P_{c, i}$ as the power level in reception, transmission and idle state on the cellular link of mobile device, respectively.

- $t_{c, r x}, t_{c, t x}, t_{c, i}$ as the time that the mobile device staying in reception, transmission and idle state on the cellular link, respectively.

The total energy consumption of a mobile device is

$$
E_{N o C o o p}=t_{c, r x} P_{c, r x}+t_{c, t x} P_{c, t x}+t_{c, i} P_{c, i}
$$

The packet size is assumed as constant and its reception time on the cellular link is one time unit.

Average energy consumption of one valid packet reception is given by

$$
E_{A R Q}=\underbrace{(1+f N \gamma \alpha)}_{t_{c, r x}} P_{c, r x}+\underbrace{f \gamma \beta \alpha}_{t_{c, t x}} P_{c, t x}+\underbrace{f \gamma \beta \alpha(N-1)}_{t_{c, i}} P_{c, i}
$$

where

- $N$ is the number of mobile devices in the network

- $\alpha$ is packet loss rate which includes both packet error and loss.

- $\gamma$ equal to $\frac{1}{1-\alpha}$, which is induced by considering the loss possibility of retransmitted packet

- $\beta$ is the ratio of the NACK size to the data packet size.

- $f$ is defined as the uncorrelated factor

- $t_{c, r x}$ consists of two parts: the time for an original packet reception, i.e., one time unit, and the time for receiving the retransmission of this packet which depends on the probability of $\mathrm{N}$ mobile devices losing this packet, the probability of the retransmitted packet loss and the uncorrelated factor of the lost packet.

- $t_{c, t x}$ is the time that the mobile device sending retransmission request, which depends on the NACK message size, the probability of the mobile device fails to receive the original packet and the retransmitted packet, and also the uncorrelated factor. Uncorrelated factor is included here, because if other mobile devices also lose the same packet and one of them has already sent a request, this mobile device does not need to send request once more.

- $t_{c, i}$ is the time that other mobile devices sending retransmission request.

The meaning behind the uncorrelated factor is that the mobile device that loses packets can recovery them without sending NACKs by itself due to the suppression NACKs scheme of SRM. In SRM specification, the mobile device that detects packet loss waits for a random time then sends NACKs. The reason is that the lost packets of each mobile device are correlated to some extend.

\section{B. Layered FEC and Integrated FEC Schemes}

In this section, we derive the energy consumption for layered FEC and integrated based on $E[M]$ (the average number of transmissions required to transmit a packet reliably to all receivers, namely, the average number of receptions a mobile device receiving a packet). $E[M]$ has been derived in [4] (Eq. 4 in [4] for layered FEC and Eq. 5,6,7 in [4] are for integrated FEC II). For the heterogeneous reception conditions, $E[M]$ can be calculated by Eq. 8,9 in [4]. Then using the same energy calculation methodology described in Subsection A., the energy consumption of layered FEC or integrated FEC II ${ }^{1}$ scheme can be expressed as

$$
E_{F E C}=\underbrace{E[M]}_{t_{c, r x}} P_{c, r x}+\underbrace{(E[M]-1) \beta}_{t_{c, t x}} P_{c, t x}+\underbrace{(N-1)(E[M]-1) \beta}_{t_{c, i}} P_{c, i}
$$

\section{COOPERATIVE RETRANSMisSiOn STRATEGY}

The idea of cooperative retransmission strategy is based on the cellular controlled peer-to-peer network architecture [2]. Multiple mobile devices (MD) located in each others' proximity can form a cooperative cluster. The MDs within a cluster can communicate directly over the short-range link (SRL). In contrast to SRL, the link between mobile devices and the access point is referred to as the cellular link (CL). The data rate of the SRL is much higher than the one of CL. Furthermore the power consumption on the SRL is much lower, because of the shorter distance between the transmitter and the receiver, which also contributes positively to the reliability of the SRL.

Exploiting the characteristics of the SRL, the MDs within one cluster can exchange (retransmit) the missed packets in a very short time over the SRL. It not only saves energy consumption but also reduces the error/loss recovery latency. It consequently increases the total throughput on the CL. In case that there is still unrecoverable packet error/loss within the cluster after cooperative retransmission, retransmission over cellular link will be triggered.

\footnotetext{
${ }^{1}$ The $E[M]$ of the integrated FEC II calculated by the formula given in [4] is the ideal lowest value. So in fact we underestimate the energy consumption of the integrated FEC II scheme.
} 


\section{A. Frame Structure Design on Cellular Link with TDD Mode}

We assume that in the investigated system the mobile device has a unified air interface. The mobile device works either on the CL or on the SRL, which is controlled by the AP. Thus all the MDs are synchronized by the clock of AP.

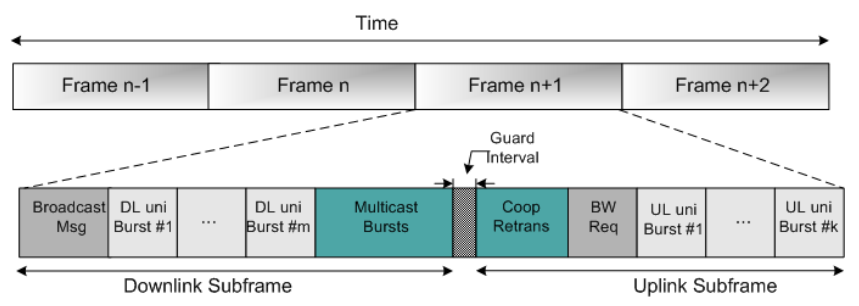

Figure 1: Frame structure in Cooperative Protocol

The investigated system uses TDD (Time Division Duplex) for the downlink and uplink transmission. The designed frame structure on the cellular link is shown in Fig. 1. All the signalling such as physical channel description, slot usage and allocation, synchronizing is included in the broadcast messages.

Hence, from the broadcast messages the subscribers know the uplink time slots allocation which includes the cooperative retransmission starting time and its duration. The time allocation for the cooperative retransmission is adjustable by the AP, according to the number of multicast service subscribers and the number of cooperative mobile devices. The time allocation information helps the MDs in a cluster to work synchronously. It is necessary to point out that the mobile device is also capable to recover error/loss stand alone when it is too far away from any cluster to cooperate with other mobile devices. The cooperative retransmission is optional for mobile device.

\section{B. Design Cooperative Retransmission Protocol on the Short Range Link}

We advocate a novel protocol design for the cooperative retransmission, which is done during cooperative retransmission slots.

First of all we assume that the cooperative retransmission is done once every $M$ multicast packets and there are $N$ mobile devices in one cluster. All the mobile devices within a cluster have the short-range links of the same data rate connected with each other. The cluster has efficient membership maintenance system. This protocol uses a token packet which is composed of Loss Packet Matrix, the Complete Reception Bit and the option field ${ }^{2}$. Due to the wireless channel independency between the mobile devices and the access point, the probability of $K$ ( $K=2 \sim 4$ ) mobile devices lose the same packet is very low. Based on the important observation, the retransmission task is usually assigned to $K$ mobile devices which are called as primary mobile devices. If the primary mobile devices are not

\footnotetext{
${ }^{2}$ The Complete Reception Bit (CRB) and the option field are used for the bad case. The CRB is initialized as " 0 " and option field is NULL. The last primary mobile device can check whether the primary mobile devices can recover all the missed packets within the cluster. If yes, the CRB is marked as " 1 "; otherwise the CRB is kept as "O". Then once any auxiliary mobile device has the correct copy of the missed packets, it will mark the CRB as "1" and insert its ID in the option field.
}

able to recovery all the lost packets, the remaining mobile devices in the cluster, called auxiliary mobile devices can help to complete recovery. Due to retransmission is done periodically, the next $K$ mobile devices in this round will work as primary mobile devices in the next round. The first mobile device in this round works as primary mobile device again in the $\lfloor N / K\rfloor$ th round.

The proposed local retransmission scheme is illustrated by an example. We assume there are four mobile devices in one cluster and two mobile devices work as primary mobile devices in each round. The cooperative retransmission consists of two procedures. The first procedure is counting all the missed packets within the cluster by marking in the Lost Packet Matrix (LPM), which is shown in Fig. 2. If mobile device $j$ loses the $i$ th packet, it marks the bit $\operatorname{LPM}(j, i)$ as bit "1", otherwise marks it as "0". A special row in the LPM is Lost Packet Information (LPI) vector which indicates all of the lost packets within the cluster. A mobile device inserts its own packet reception information and marks the LPI vector when it receives the token packet.

As soon as the first mobile device receives the token packet

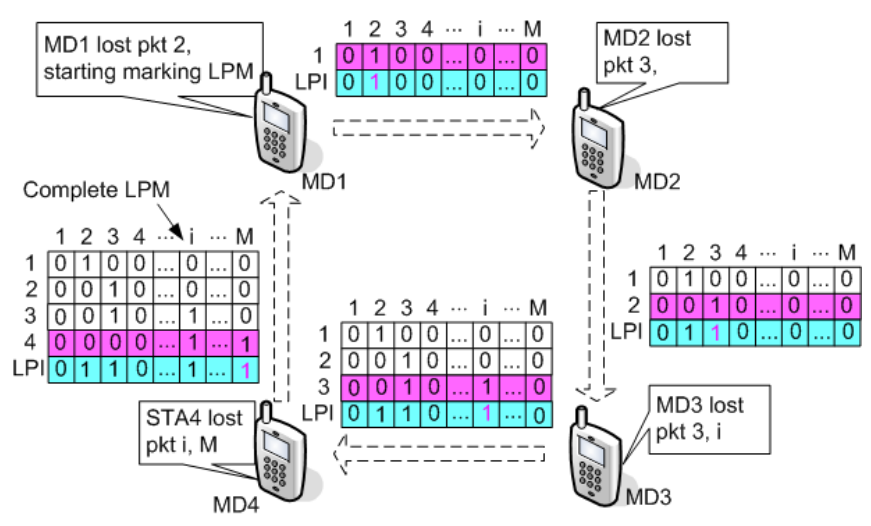

Figure 2: Marking Lost Packet Matrix procedure

with the complete LPM from the last mobile device, it starts local retransmission which is shown in Fig. 3. Assuming there are $L$ induplicated lost packets within the cluster. According to the information in LPM, the primary mobile device in the cluster always first retransmits the packets which it has but other primary mobile devices' loses. And each primary mobile device shares the retransmission task of sending at least $\lceil L / K\rceil$ packets. Each primary mobile device resets the index bits of the retransmitted packets as "0" in the LPI vector and passes the token packet to another primary mobile device after completion of its duty. After last primary mobile device finishes its retransmission duty, it uses the information from the CRB and the option field to guarantee to complete error recovery.

Furthermore, a simple compression algorithm is used to compress LPM from $N+1$ rows to $K+1$ rows. The compressed LPM of the above example is shown as Fig. 4. The idea is that the auxiliary mobile device can mark the lost packet on a random row but with a specific column index, instead of having an individual row to indicate its lost packets. 


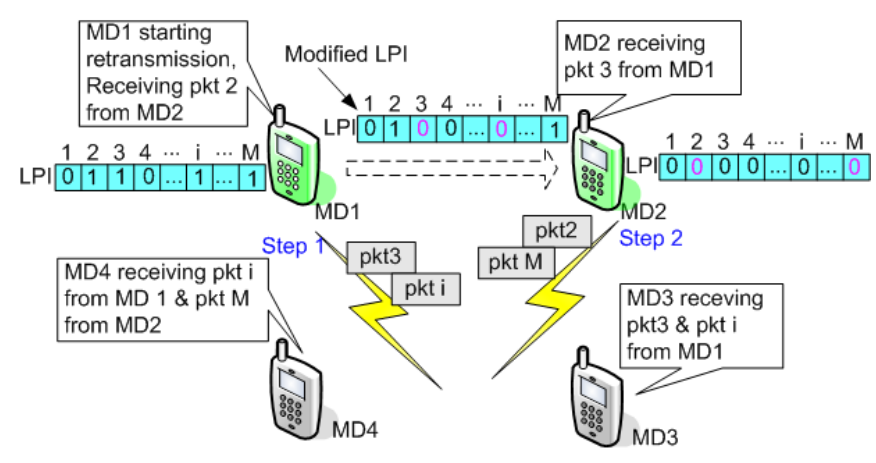

Figure 3: Local retransmission procedure (here MD1 and MD2 are primary mobile device, i.e., retransmitters)

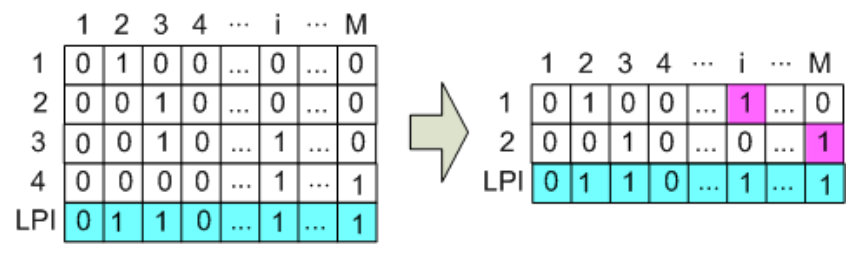

Figure 4: Compressed Lost Packet Matrix for the example

This cooperative retransmission scheme is very fair for all the mobile devices within a cluster. Furthermore, it highly meets the timely reciprocity requirements [2] of the designing principle for cooperative wireless networks. Every cooperating party should see his benefits in doing so within the shortest possible delay [2]. The delay of the feedback benefit in the proposed scheme is only at the order of seconds, which can be regarded as nearly instantaneous reciprocity.

\section{Energy consumption by Cooperative Retransmission Pro- tocol}

In reality, the unrecoverable packet loss probability is very low after cooperative retransmission. Cellular retransmission is seldom needed. Furthermore, as we know SRL is very reliable, hence we assume no packet loss over SRL. We define:

- $P_{s r, r x}, P_{s r, t x}$ and $P_{s r, i}$ as the power level in reception, transmission and idle state on the short-range link of mobile device, respectively.

- $t_{s r, r x}, t_{s r, t x}$ and $t_{s r, i}$ is the time that the mobile device staying in the corresponding states.

Now the total energy consumption of MD consists of two parts: $E_{c}$ the energy consumption on the CL and $E_{s r}$ that on the SRL. Based on the assumption, it is clear that a mobile device only works on its cellular interface when it receives the original data packets; and it works on its short-range interface during the remaining time.

$$
E_{C o o p}=\underbrace{t_{c, r x} P_{c, r x}}_{E_{c}}+\underbrace{t_{s r, r x} P_{s r, r x}+t_{s r, t x} P_{s r, t x}+t_{s r, i} P_{s r, i}}_{E_{s r}}
$$

$E_{s r}$ consists of: the energy consumption used for marking LPM and local retransmission. During one marking LPM procedure, each MD receives and transmits the LPM once. The
Table 1: Parameters assumption for Analysis

\begin{tabular}{|c|c||c|c|}
\hline Notation & Value & Notation & Value \\
\hline$P_{c, r x}$ & $0.9 \mathrm{~W}$ & $P_{s r, r x}$ & $0.4 \mathrm{~W}$ \\
$P_{c, t x}$ & $2 \mathrm{~W}$ & $P_{s r, t x}$ & $1 \mathrm{~W}$ \\
$P_{c, i}$ & $0.04 \mathrm{~W}$ & $P_{s r, i}$ & $0.04 \mathrm{~W}$ \\
$R_{c}$ & $6 \mathrm{Mbps}$ & $R_{s r}$ & $54 \mathrm{Mbps}$ \\
$\alpha$ & $2 \%$ or 5\% & $\beta$ & 0.2 \\
$\rho$ & 0.1 & $\omega$ & 9 \\
\hline
\end{tabular}

MD can stay in idle state during the remaining time of one marking LPM period. So the energy overhead due to marking LPM for each packet is denoted by $\delta$ which is given by

$$
\delta=\frac{\rho}{\omega}\left(P_{s r, t x}+P_{s r, r x}+(N-2) P_{s r, i}\right) / M
$$

where, $\rho$ is the ratio of the average token packet size to data packet size; and $\omega$ is the data rate ratio of SRL to CL.

The average energy overhead for each packet on each MD due to local retransmission is derived as following. As there are $K$ primary mobile devices in a local retransmission, each MD takes retransmission task every $N / K$ local retransmissions. Hence, we can sum up one mobile device's energy consumption on the short-range interface during the consecutive $N / K$ local retransmissions. Then we average the sum by $N / K$ and $M$ (local retransmission is done once for every $M$ multicast packets). So $E_{s r}$ can be expressed by

$$
E_{s r}=\frac{E_{p r i}+\left(\frac{N}{K}-1\right) E_{a u x}}{\frac{N}{K} M}+\delta
$$

Where, $E_{\text {pri }}$ and $E_{a u x}$ is the energy consumption of a mobile device working as primary or auxiliary mobile device in one local retransmission. Their expressions are given by

$$
\begin{gathered}
E_{\text {pri }}=\frac{L / K}{\omega} P_{s r, t x}+\frac{L / K}{\omega}(K-1) P_{s r, r x} \\
E_{a u x}=\frac{L}{\omega} P_{s r, r x}
\end{gathered}
$$

The explanation of $E_{p r i}$ and $E_{a u x}$ expression is: after AP transmitting $M$ multicast packets, the number of packets lost at one mobile device is equal to $M \alpha$; the sum of lost packets at all mobile devices in one cluster is $N M \alpha$; due to correlation of the lost packets, the number of the unduplicated lost packets within one cluster is $L=f N M \alpha$.

\section{COMPARISON OF ENERGY CONSUMPTION}

This section compares the energy consumption of the cooperative retransmission strategy against the non-cooperative ones. Table 1 lists the variables for the energy analysis. The parameters are taken from [2].

Fig. 5 and Fig. 6 compare the energy consumption between the non-cooperative and the cooperative strategies under condition of homogeneous and heterogeneous packet loss rate at the receivers, respectively. It clearly shows that the energy consumption of the cooperative strategy is quite stable with the 
number of mobile devices increasing in both homogeneous and heterogeneous packet loss rate cases. However the energy consumptions of the layered FEC and the ARQ (SRM) schemes increase dramatically when the number of the mobile devices increases. It is obvious that higher packet loss rate leads to more energy saving. In these two figures, we can also see the effect of ARQ (SRM) suppression scheme. For example, due to uncorrelated factor deceasing with the number of the mobile devices increasing, the increasing trend of energy consumption of ARQ (SRM) decreases with mobile devices increasing. Moreover, in Fig. 6, the two ARQ (SRM) curves are overlapped because the suppression scheme in SRM reduces the duplicated retransmission.

The integrated FEC II has as good energy consumption as the cooperative retransmission scheme when the receivers have the homogeneous packet loss. But the cooperative retransmission scheme outperforms integrated FEC II under the heterogeneous packet loss rate assumption. For instance, it can be seen in Fig. 6 that in the scenario of 128 mobile devices with about six high packet loss mobile devices in one cluster, the cooperative scheme can save energy up to $40 \%$.

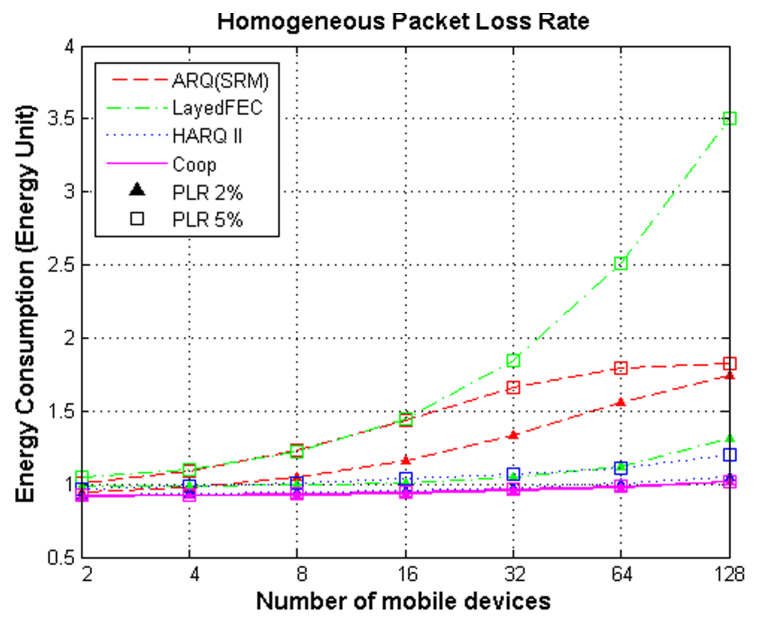

Figure 5: Energy consumption comparison of different error recovery schemes in homogeneous packet loss rate scenario

\section{CONCLUSION}

In this paper, we propose a novel and generic cooperative retransmission scheme for the wireless reliable multicasting based on CCP2P network architecture. It exploits the higher data rate, better reliability and lower power consumption characteristics of the short-range link and can recover almost all packet error/loss locally in a very short time. It can reduce energy consumption and minimize packet loss recovery latency. It is robust to not only homogeneous but also to heterogeneous channel conditions. Comprehensive comparison and analysis of energy consumption between the cooperative and the noncooperative strategies have been given. The analysis results show that the proposed cooperative retransmission protocol is more efficient and suitable for the reliable multicast services than the non-cooperative ones.

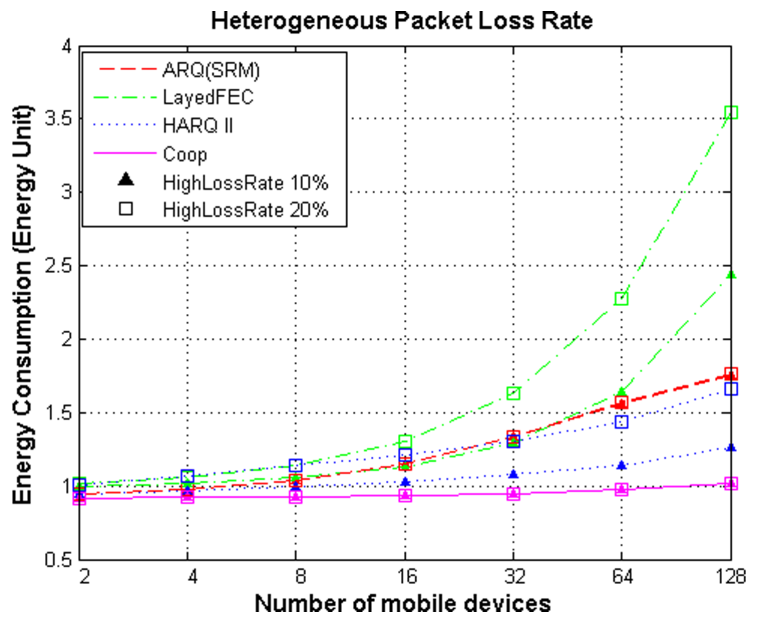

Figure 6: Energy consumption comparison of different error recovery schemes in heterogeneous packet loss rate scenario (The high packet loss is $10 \%$ or $20 \%$. There are $5 \%$ high packet loss mobile devices in the cluster. The rest of mobile devices has $2 \%$ packet loss rate.)

\section{REFERENCES}

[1] J.William Atwood. A classification of reliable multicast protocols. IEEE Nextwork, 18:24-34, 2004.

[2] Frank H.P. Fitzek and Marcos D.Katz, editors. Cooperation in Wireless Networks: Principle and Applications. ISBN-10 1-4020-4710-X. 2006.

[3] M. Yamamoto. Multicast communication in next-generation internet. 10th IFAC/IFORS/IMACS/IFIP Symposium on Large Scale Systems: Theory and Applications, pages 639-645, July 2004.

[4] Don Towsley Jorg Nonnenmacher, Ernst W. Biersack. Parity-based loss recovery for reliable multicast transmission. IEEE/ACM Trans. on Networking, 6(4), August 1998.

[5] Li-wei H. Lehman, Stephen J. Garland, and David L. Tennenhouse. Active reliable multicast. Proceedings - IEEE INFOCOM, 2:581-589, 1998.

[6] R. Govindan D. Estrin P. Radoslavov, C. Papadopoulos. A comparison of application-level and router-assisted hierarchical schemes for reliable multicast. IEEE/ACM Trans., 12:469-482, June 2004.

[7] E.R.Berkekamp. Algebraic Coding Theory. McGrawHill, 1968.

[8] F.J.MacWilliams and N.J.A. Sloane. Theory of Error Correcting Codes. 1977.

[9] D.M.Mandelbaum. Adaptive-feedback coding scheme using incremental redundancy. IEEE Trans. Infom. Theory., pages 388-289, May 1974.

[10] I. Maric and R.D. Yates. Cooperative multicast for maximum network lifetime. IEEE Journal on Selected Areas in Communications, 23(1):127-135, 2005.

[11] Katia Obraczka. Multicast transport protocols:a survey and taxonomy. IEEE Communication Magazine, January 1998. 